Die Laokoon-Debatte: Kunstreflexion und Medienkonkurrenz im 18. Jahrhundert

Schneider, Sabine

Posted at the Zurich Open Repository and Archive, University of Zurich ZORA URL: https://doi.org/10.5167/uzh-100711

Book Section

Published Version

Originally published at:

Schneider, Sabine (2014). Die Laokoon-Debatte: Kunstreflexion und Medienkonkurrenz im 18. Jahrhundert. In: Benthien, Claudia; Weingart, Brigitte. Handbuch Literatur Visuelle Kultur. Berlin: De Gruyter, 68-85. 


\subsection{Die Laokoon-Debatte: Kunstreflexion und Medienkonkurrenz im 18. Jahrhundert}

Sabine Schneider

\section{Krise der Repräsentation}

Die von der aktuellen Bildwissenschaft gestellte Diagnose einer ,ikonischen Wende' sieht im Zeitalter der Moderne das kulturelle Leitmedium der Schrift in besonderer Weise mit der Konkurrenz von Bildwelten konfrontiert (vgl. Boehm 1994). Bereits für die Anfänge der modernen episteme im 18. Jahrhundert hat diese These Plausibilität. Die Bilderfrage ist in der kulturgeschichtlichen Forschung eng mit der Modernedebatte verknüpft. Die Genese der Makroepoche der Moderne in der Umbruchszeit zwischen Spätaufklärung und Romantik lässt sich als Doppelbewegung von krisenhaftem Traditionsabbruch und reflexivem Neuansatz an medialen und semiotischen Fragen beobachten. So hat die jüngere Romantikforschung die dialektische Verschränkung von „kühnen und spielerischen Anfangsexperimenten“ mit einer „Medienkrise ersten Ranges“ konstatiert, „die im Feld der neu entwickelten wissenschaftlichen und künstlerischen Medien aufbricht und dabei deren Verwendung im Akt des Erkennens und des Bildens nachhaltig beeinflusst und umstrukturiert“ (Neumann und Oesterle 1999, 9).

Es ist symptomatisch für das ausgehende 18. Jahrhundert, dass sich das Bewusstsein für mediale Eigenlogiken zum einen innerhalb des ästhetischen und kunsttheoretischen Diskurszusammenhangs artikuliert und somit Medienreflexion als Konkurrenzverhältnis zwischen den Künsten betreibt, zum anderen seine Sensibilisierungen vor allem in Auseinandersetzung der Sprachkunst mit der bildenden Kunst gewinnt (vgl. Pethes 2002; Pfeiffer 1999): „Malerei und Dichtung sind die Paradigmen, an denen das 18. Jahrhundert die Mediengebundenheit der ästhetischen Erfahrung entdeckt hat“ (Stierle 1984, 23). Dass sich mediale Neuerungskrisen und deren reflexive Aufarbeitung im 18. Jahrhundert gerade an der Mediendifferenz zwischen Sprache und Bild artikulieren (und nicht etwa an der zur Musik), hat mit der internen Logik des Aufklärungsprozesses zu tun, aber auch mit Veränderungen im Kunstsystem und in den kulturellen Praktiken der Kunstproduktion sowie -rezeption. Gründe für diese Entwicklungen können nicht monokausal aufgefunden werden. Sie sind vielfältig und entstammen unterschiedlichen Bezugssystemen.

Eines dieser in der zweiten Jahrhunderthälfte in die Krise geratenen Systeme ist die rationalistische Logik und Sprachtheorie der Schulphilosophie. Hier ist 
die Dialektik von Krise und Neuerung besonders augenfällig. Die von der Aufklärungsforschung der letzten Jahrzehnte unter dem Stichwort ,Rehabilitierung der Sinnlichkeit' verhandelte Aufmerksamkeit auf die Eigenlogiken des Bildlichen und Visuellen und deren Abgrenzung zum Sprachlich-Diskursiven ist die Folge einer radikalisierten Aufklärung, der ihr eigenes im Zuge eines Rationalisierungs- und Abstraktionsprozesses durchgesetztes Bildverbot zum Problem geworden ist. Demgegenüber macht sich zunehmend ein anthropologisches Bildbedürfnis geltend. Die Konsequenzen sind einerseits die Herausbildung der Ästhetik als eigenständige Erkenntnisform des Sinnlichen, andererseits veränderte poetologische Konzepte in der Literatur. Deren besondere Herausforderung zur Ausbildung „neuer, sprachlich konstituierter Bildkonzepte“ liegt darin, dass ihr eigenes, sprachliches Medium das Leitmedium für die aufklärerische Austreibung der Bilder aus Denken und Sprechen dargestellt hatte und nun einer Selbstkorrektur von innen bedurfte (vgl. Schneider et al. 2001, 9). Die Literatur übernimmt das von der Philosophie marginalisierte Geschäft der ,Bildkritik ${ }^{\star}$ - in einem Kantischen Sinn als Bildreflexion und Auslotung ihrer Möglichkeiten und Grenzen (vgl. Simon 2009). Die neue Disziplin der Ästhetik mit ihrem Bezugssystem der aufgeklärten Erkenntnislehre und Logik auf der einen Seite und die aufgeklärte Poetik mit ihrem Bezugssystem der antiken Rhetorik auf der anderen Seite haben also den Plausibilitätsverlust der Rationalität und die sich daraus ergebenden Chancen für eine Neubewertung des Bildlichen zu verhandeln. Analogon rationis lautet die Formel für diese Neubewertung in der Ästhetik, welche die Sinnlichkeit als eigenständige Erkenntnisform der Rationalität gleichberechtigt an die Seite stellt. Ut pictura poesis ist die entsprechende Formel der Poetik, die beide Schwesterkünste, Poesie und bildende Kunst, auf dasselbe Prinzip der Nachahmung verpflichtet. Zwischen beiden Feldern bestehen vielfältige argumentative Verbindungen, die vermittelt sind über anthropologische Theorien der Seelenvermögen, über die Leistungen von Anschauung, Einbildungskraft und Abstraktion sowie deren Beziehung zu Zeichenprozessen. Anthropologische, ästhetische und semiotische Argumentationen gehen Hand in Hand.

Die Aufmerksamkeit der Spätaufklärung auf die leibnahen niederen Seelenvermögen nobilitiert die Anschauung. Sie wird in der Terminologie der Schulphilosophie bestimmt als cognitio clara et confusa (vgl. Leibniz 1999 [1684], 585-586). Als nicht im Sinne der analytischen Abstraktion distinkte, sondern im Sinne konturierter sinnlicher Erkenntnis klare und prägnante Wahrnehmung ist sie nun nicht mehr nur untergeordnetes Hilfsinstrument für die abstrakte Vernunft wie in der Frühaufklärung. Der sinnlichen Erkenntnis wird als „analogon rationis“ (Baumgarten 2007 [1750-1758], 21), so die Formulierung des Begründers der Ästhetik, Alexander Gottlieb Baumgarten, eine fundamentale und eigenständige Bedeutung für die Erfassung der Wirklichkeit zugebilligt (vgl. Adler 1988). 
Damit wird die Ästhetik im Sinne von aisthesis (sinnliche Wahrnehmung) der Logik zur die Seite gestellt. Verbunden damit ist die Aufwertung von Kriterien wie ,Prägnanz‘ als Einheit in der Mannigfaltigkeit der Sinnesdaten sowie von sinnlicher Fülle, die den Abstraktionen des rationalen Denkens gegenüber ins Spiel gebracht werden kann (vgl. Adler 1998).

Die Neubewertung der sinnlichen Erkenntnis hat Verunsicherungspotential, das mit den Hierarchien des erkennenden Denkens auch die Verbürgtheit der adaequatio von Welt und Erkenntnis erschüttert und die Repräsentationsfunktion der Zeichensysteme infrage stellt. Die historische Einsicht in die Eigenlogiken des Bildlichen ist somit durchaus nicht ohne Risiko. Eine Erkenntniskrise und eine semiotische Krise gehen damit einher. Erstere artikuliert sich innerhalb der Ästhetik und der Psychologie in der Neubewertung der Einbildungskraft, die von einem rein reproduktiven Vermögen der mentalen Übersetzung visueller Eindrücke zu einem selbsttätigen, produktiven Vermögen der Neuschöpfung von bildlichen Vorstellungen ermächtigt wird. Damit findet in den Jahren zwischen der rationalistischen Vermögenspsychologie Christian Wolffs am Anfang und der transzendentalen Subjektphilosophie Immanuel Kants am Ende des 18. Jahrhunderts ein Paradigmenwechsel statt. Das freigesetzte Vorstellungsbild verliert mit dem einheitlichen Vernunftgrund auch den Gegenstandsbezug und wird zur Vorstellungsart subjektiviert (vgl. Kant 1977b [1790], 294). Damit verbunden ist eine „Rhetorisierung des Denkens“ (Gaier 2001, 19), die - vermittelt über die Theorie der assoziativen Verknüpfung über Kontiguitäten - lange vor Friedrich Nietzsche die Transparenz des Denkens infrage stellt und das „Denken als Bildprozess“ (ebd., 27) ohne festen Referenzpunkt fasst. Die Literatur der Klassik und Romantik am Jahrhundertende wird diese Theorie mentaler Bildlichkeit aufgreifen und daraus ein Bewusstsein für den Eigensinn des literarisch generierten Bildes entwickeln, das sich aus seiner intellektualistischen Repräsentationsfunktion löst und als konfiguratives Gebilde eine eigene, poietisch erzeugte, Wirklichkeit darstellt, (vgl. Pfotenhauer 2000, 7-9). Die destabilisierende Wirkung dieser Neubewertung zeigt die zeittypische Pathologisierung der produktiven Einbildungskraft als Komplementärform ihrer Entmimetisierung. Der ästhetischen Freisetzung der Einbildungskraft wird eine Warnung vor ihren Chimären und Kopfverrückungen beigesellt (vgl. Kant 1977a [1798], 476; Jakob 1791, 297).

Mit der rationalistischen Erkenntnistheorie war eine Semiotik des arbiträren Zeichens verknüpft, die das distinkte Denken und seine Abstraktionsoperationen in eine Theorie der sprachlichen Repräsentation münden ließ und die Einheit von Gegenstand, Denken und Sprache garantierte. Vom Plausibilitätsverlust der schulphilosophischen Erkenntnistheorie betroffen ist auch ihr rationalistisches Zeichenkalkül. Dem abstrakten, durch Konvention gesetzten und auf Merkmalsabstraktionen beruhenden ,willkürlichen Zeichen' - und damit der Sprache - 
wird nicht mehr zugetraut, die sinnliche Wirklichkeit der Dinge zu erfassen. Die im Kontext der Sprachkritik der Aufklärung geprägte zeittypische Rede vom ,toten Buchstaben' nach Paulus (2 Kor 3,1-6) ist dafür ebenso ein Indikator wie die Hochschätzung des ,natürlichen Zeichens‘. Diese aus dem Sensualismus stammende zeichentheoretische Unterscheidung zwischen den arbiträren konventionellen Zeichen der Wortsprache und den entweder ikonisch, expressiv oder kausal motivierten Zeichen, wie sie den Bildern oder auch dem expressiven unwillkürlichen Körperausdruck zugeschrieben werden, führt Moses Mendelssohn 1757 in die deutschsprachige Debatte ein (vgl. Mendelssohn 1986 [1757], 182). Die Konsequenzen für die Medienkonkurrenz im 18. Jahrhundert und den Legitimierungszwang der Literatur liegen auf der Hand. Die semiologische Unterscheidung der Zeichentypen stellt die Voraussetzung dar für die Konzeptionen des ästhetischen Zeichens in der Kunstreflexion in Abgrenzung zum arbiträren Zeichen der Aufklärungssemiotik. Sowohl die Konjunktur des ,natürlichen Zeichens' in der Kunsttheorie wie auch die Redeform der Überbietung, derzufolge die Sprache wie bei Karl Philipp Moritz oder Wilhelm Heinrich Wackenroder eine ,höhere Sprache‘ zu sein hat, die das ästhetische Zeichen mit metaphysischen Signaturmodellen auflädt oder sich bei der Renaissance-Hieroglyphik rückversichert (vgl. Moritz 1995 [1788], 373-374; Wackenroder 1991 [1796]), zeugen von dieser Verunsicherung (vgl. Pfotenhauer 1991).

Unmittelbar relevant für die Kunstreflexion und das Verhältnis der Künste wird die Krise der Sprache schließlich in der semiotischen Präzisierung des seit der Antike in der Poetik etablierten Unsagbarkeitstopos, der besagt, die Schönheit des Kunstwerks lasse sich nicht in Worte fassen, und der in der Kunstliteratur der Klassik und Romantik eine neue Konjunktur erfährt. In der sprachlichen Beschreibung eines Werks der bildenden Kunst verschärft sich der TautologieVorwurf an das in seinen Repräsentationen befangene Sprachsystem, dem es an sinnlicher Konkretion und Motiviertheit mangele. Infolgedessen könne, so der konsequenteste Vertreter einer Nichtübersetzbarkeit der Bildkunst in Sprache, Karl Philipp Moritz, das Schöne der bildenden Kunst „nur mittelbar durch Worte beschrieben werden [...], die oft erst einen sehr weiten Umweg nehmen, und manchmal eine Welt von Verhältnissen in sich begreifen müssen, ehe sie auf dem Grunde unseres Wesens dasselbe Bild vollenden können, das von außen auf einmal vor unserm Auge steht“ (Moritz 1995 [1788], 379). 


\section{Medienkonkurrenz der Künste: Das „Laokoon-Paradigma“}

Das Verhältnis von Poesie und Bildkunst steht nicht nur in der Textgattung der Kunstliteratur neu zur Disposition und zwingt insbesondere die Sprachkünste zur Reflexion ihrer medialen Vermitteltheit in Auseinandersetzung mit der Leistung anderer Medien. Es muss auch innerhalb der traditionellen Poetik das Verhältnis von Sprache und Bild, wie es unter Berufung auf Horaz in den Poetiken seit der Antike unter die Formel ut pictura poesis gefasst worden war, neu ausgelotet werden (vgl. 2.2 BERNDT und 3.1 WETZEL). Im Schnittpunkt beider Begründungszusammenhänge - der Kunstliteratur im Gefolge Johann Joachim Winckelmanns und der sich auf antike Autoritäten berufenden Aufklärungspoetik - steht Gotthold Ephraim Lessings Abhandlung Laokoon oder Über die Grenzen der Malerei und Poesie (1766), die als erste moderne Formulierung einer Medienästhetik gelten kann. Die Bedeutung dieser Schrift liegt dabei weniger in der Originalität der einzelnen Argumente - Lessing fasst vielmehr die Diskussion zur Medienbezogenheit der ästhetischen Erfahrung zusammen und fokussiert sie neu - als in der Radikalisierung der medientheoretischen Perspektive auf die Kunst und im Willen zu kategorischen Grenzziehungen zwischen den Einzelkünsten. „Lessings Laokoon ist die erste konsequente Ausarbeitung der Medienästhetik, die in der Natur des Mediums die Natur der Kunst begründet sein läßt. So wird hier, was bisher nur eine technische Voraussetzung zu sein schien, zu einem Moment der Kunst selbst“ (Stierle 1984, 38).

Wie sehr diese konsequente medienästhetische Perspektive auf die Künste die Veränderungen in der Kunstauffassung auf den Punkt brachte, zeigt die beispiellose Rezeptionsgeschichte von Lessings Schrift. Die Provokation seiner Radikalisierung fordert nicht nur in den folgenden drei Jahrzehnten alle maßgeblichen Vertreter der ästhetischen Theoriebildung zu einer Entgegnung oder Fortführung heraus, angefangen mit Johann Gottfried Herder über Wilhelm Heinse, Moritz, Friedrich Schiller, Aloys Hirt bis hin zu Johann Wolfgang von Goethe (vgl. Pfotenhauer et al. 1995, 530-559). Die ,Laokoon-Debatte‘ dauert noch das ganze 19. Jahrhundert über und im Grunde bis heute an: „Laokoon und kein Ende“ (vgl. Koebner 1989). An ihr zeigt sich der paradigmatische Zusammenhang zwischen der Bewusstwerdung von Mediengrenzen zwischen den Künsten und der Herausforderung, diese scharf gezogenen Grenzen wieder zu überschreiten. Wann immer die Künste die Grenzen ihres Materials zu überschreiten beanspruchen, die Literatur gegen das Sukzessivitätsgebot des Narrativen aufbegehrt oder die bildende Kunst die Zeit und das Unsichtbare für sich entdeckt, steht Lessings Medienästhetik neu zur Disposition. „Das Laokoon-Paradigma“, wie die jüngere Forschung im Anschluss an Lessings Schrift die Dialektik von Grenzziehung und Entgrenzung zwischen den Künsten genannt hat, ist zum Katalysator für 
die Medienreflexivität der Künste in der Moderne avanciert (vgl. Baxmann et al. 2000).

Ausgangspunkt ist das von den Barock- wie Aufklärungspoetiken transportierte ut pictura poesis-Gebot. Unter Berufung auf Horaz verpflichtet es die Poesie auf das Vorbild der Malerei und umgekehrt die Malerei auf das kulturelle Schriftwissen der Poesie. Für die Malerei implizierte dieses Gebot einen Intellektualismus, der sich noch in Winckelmanns Programmschrift Gedanken über die Nachahmung der griechischen Werke in Malerei und Bildhauerkunst (1755) geltend macht - in der Formulierung, der Pinsel des Malers müsse ,im Verstand getunckt seyn“ (Winckelmann 1995b [1755], 50). Dabei erfuhr eine normative Verfestigung, was in den Malereitraktaten von der italienischen Renaissance bis zur französischen Klassik einst einem legitimatorischen Diskurs der Malerei gegolten hatte (siehe dazu 3.1 Wetzel). Die artistische Malerei sollte, so in Leonardos Vorstoß im Wettstreit der Künste, in den Stand einer ars liberalis erhoben und vom Verdikt des bloßen Handwerks befreit werden. Diese Nobilitierung konnte nur durch die Berufung auf die kanonischen rhetorischen Autoritäten der Antike erfolgen. Die in Plutarchs De gloria Atheniensium (2012 [1. Jh. n. Chr.], 603-604) überlieferte Anekdote, wonach der griechische Maler Simonides die Malerei eine ,stumme Poesie‘ und die Poesie eine ,sprechende Malerei‘ genannt habe, gehört seit der Renaissance ebenso zum Bestand dieser wechselseitigen Bezugnahme wie die Stelle aus Horaz' De arte poetica (Horaz 1967 [ca. 14 v. Chr.], 250-251, V. 361-365), die seit dem 17. Jahrhundert zur normativen Formel erstarrte (vgl. Buch 1972, 20-22; Willems 1989, 216-295). Ontologische Voraussetzung für diese Formel war die unangefochtene Legitimität des aristotelischen Mimesis-Gebots, das bis in die zweite Hälfte des 18. Jahrhunderts mit dem Repräsentationsparadigma der rationalistischen Logik und Semiotik vereinbar war. Alle Künste waren über das Gebot der Nachahmung auf denselben ontologischen Grund verpflichtet, was das ästhetische Zeichen auf seine Repräsentationsfunktion reduzierte und ihm einen rein instrumentellen Status zum Transport der Merkmale des Bezeichneten zugestand. Weder der mediale Anteil an der Sinnkonstitution noch die Eigenart des Bildlichen - geschweige denn dessen Ausdifferenzierung - konnten im Horizont dieser rationalistischen Ontologie in den Blick geraten. ,Bild“ oder ,Gemälde، bezeichnet in der Semantik des 18. Jahrhunderts ununterschieden die Repräsentation eines Gegenständlichen entweder im Vorstellungsbild oder in der künstlerischen Realisation und dabei wiederum unterschiedslos im materiellen Gemälde auf einer Leinwand wie in der poetischen Beschreibung.

In den Poetiken des 18. Jahrhunderts artikuliert sich dieser Zusammenhang in der Rede über die ,malende Poesie‘, die dann zum Zielpunkt von Lessings Attacke werden sollte. Theoretisch ausformuliert wurde diese Poetik von Johann Jakob Bodmer und Johann Jakob Breitinger. Dabei ist deren noch der rationalis- 
tischen Repräsentationstheorie verpflichteter, mimetischer Ansatz keineswegs spannungsfrei. Auch für diese frühaufklärerische Poetik ist die im Bereich der sensualistischen Ästhetik bereits Anfang des Jahrhunderts formulierte Zeichentheorie mit ihren wirkungsästhetischen Implikationen zur Herausforderung geworden, auf die es zu reagieren gilt und die den Wettstreit der Künste erneut aufruft: Jean-Baptiste Dubos hat in seinen wirkungsmächtigen Réflexions critiques sur la poésie et sur la peinture (1719) den Begriff der Mimesis konsequent wirkungsästhetisch aufgefasst und ihn auf die durch die Nachahmung vermittelten Emotionen bezogen. Da der Fokus solcherart von der Repräsentationslogik des Kunstwerks (also dessen Objektbezug in der durch Zeichen repräsentierten Nachahmung) auf die ästhetische Erfahrung (und damit auf die Vermittlung zwischen Werk und Rezeptionsakt) verschoben wird, tritt erstmals die Eigenart des Medialen als Vermittlung dieser Erfahrung in den Blick. Erst diese ermöglicht eine mediale Ausdifferenzierung der Künste.

Bei Dubos artikuliert sich das neue mediale Bewusstsein wie nach ihm bei Lessing als Zeichentheorie: Dass die Malerei sich der signes naturels bedient, welche durch die unmittelbare Ähnlichkeit zwischen Zeichen und Bezeichnetem unmittelbare Evidenz erzeugen, sichert ihr a priori eine größere Wirkungskraft zu. Demgegenüber hat nach Dubos die Poesie mit ihren signes artificiels die komplexere mediale Vermittlungsleistung zu erbringen, was ihre Wirkung aufgrund des zeitlichen Verschiebungsfaktors und der potenzierten Prozedur der Übertragung, aber auch wegen der konventionellen Beziehung zwischen Wort und Idee, abschwächt: „Die Worte müssen erst diejenigen Ideen rege machen, deren blos willkührliche Zeichen sie sind. Darauf müssen sich diese Ideen in der Einbildungskraft in Ordnung stellen, und daselbst die Gemählde bilden, die uns rühren und interessieren sollen“ (Dubos 1760 [1719], 369). Während Dubos aus dieser Zeichentheorie eine Überlegenheit der Malerei im Paragone der Künste folgert, kehren Bodmer und Breitinger die Argumentation zugunsten der Poesie um. Die unmittelbar einsichtigen natürlichen Zeichen der Malerei sichern ihr zwar sinnliche Evidenz zu, schränken sie aber auch auf den Bereich des Sichtbaren ein. In einer Überbietungslogik ist demgegenüber die Poesie die höhere Form von Malerei: „Der Poet mahlet nicht für das Auge allein, sondern auch für die übrigen Sinnen, und er kann auch das unsichtbare sichtbar machen, er giebt dem Menschen nicht nur die vollkommenste Bildung, sondern auch die Rede [...]“ (Breitinger 1966 [1740], 19; siehe dazu auch Koschorke 1999, 282).

Mit der größeren Freiheit der durch die Poesie erzeugten Vorstellungsbilder argumentiert auch Lessing drei Jahrzehnte später - mit dem wesentlichen Unterschied, dass er den Begriff des ,Gemäldes‘ für die Poesie nun einer semantischen Kritik unterzieht und ihn in Abgrenzung zur Malerei als poetische Illusion spezifiziert. Er kritisiert die „Zweideutigkeit des Wortes“ und möchte es durch 
„Phantasie“, „Illusion“ oder - unter Berufung auf Plutarch - durch „Enargie, Träume der Wachenden“ ersetzt sehen (Lessing 1990 [1766], 113-114). Der Begriff der enargeia verweist auf das Bezugssystem der Rhetorik (vgl. 2.2 BERNDT). Er bezeichnet den Medienwechsel vom Hören zum inneren Sehen durch eine sinnlich gemachte Rede, also die rhetorische Technik der evidentia, welche die Darstellungsleistung der Einbildungskraft zur Erzeugung sinnlicher Evidenz mobilisiert. Die Umsetzung dieser Forderung für die Poesie in der sinnlich gemachten Sprache folgt dann aber in Lessings Argumentation nicht mehr der klassischen Rhetorik, sondern einer spezifisch semiotischen Argumentation und der Forderung der Ästhetik nach sinnlicher Erkenntnis: „[J]eder Zug, jede Verbindung mehrerer Züge, durch die uns der Dichter seinen Gegenstand so sinnlich macht, daß wir uns dieses Gegenstandes deutlicher bewußt werden als seiner Worte, heißt malerisch“ (Lessing 1990 [1766], 113). Lessing folgt hier der Zeichentheorie Mendelssohns, der in seiner Abhandlung Über die Hauptgrundsätze der schönen Künste und Wissenschaften (1757) eine spezifisch literarische, sprachliche Motivierung der Sprachzeichen in der Poesie fordert und dabei die Semiotik auf die sinnliche Erkenntnis der Ästhetik verpflichtet: „Das Mittel, eine Rede sinnlich zu machen, besteht in der Wahl solcher Ausdrücke, die eine Menge von Merkmalen auf einmal in das Gedächtniß zurück bringen, um uns das Bezeichnete lebhafter empfinden zu lassen als das Zeichen. Hierdurch wird unsere Erkenntnis anschauend“ (Mendelssohn 1986 [1757], 183). Die Anschaulichkeit der Dichtung ist somit schon bei Mendelssohn keine an der Malerei orientierte Gegebenheit mehr, sondern Ergebnis einer semiotischen und medialen Reflexion, die wie bei Lessing im Dienst einer Illusionstheorie steht. Um das Vergessen der Zeichen auszulösen, bedarf es Techniken der Illusionierung. Wirkungstheoretische Konzepte haben somit im Zuge der Medienreflexion die aristotelische Mimesis abgelöst.

Lessing wendet das Argument gegen die „Schilderungssucht“ (Lessing 1990 [1766], 209) in der Poesie, mit der Stoßrichtung gegen das deskriptive Lehrgedicht, für das er als Beispiel Albrecht von Hallers Die Alpen (1729) anführt (siehe dazu auch 4.6 KöHNEN). Hallers Text verfährt noch rationalistisch, indem er die Beschreibung eines morgendlichen Alpenszenarios als sukzessive Addition von Teilbeschreibungen wiedergibt, aus deren Merkmalssumme der Totaleindruck additiv zusammengesetzt werden muss. Das Nacheinander von Merkmalanalyse und begrifflicher Synthese entspricht der rationalistischen Erkenntnistheorie. Lessing führt gegen dieses zergliedernde Verfahren die zugleich wahrnehmungspsychologische und ästhetische Kategorie der ,Ganzheit‘ ins Feld. Sie wird der Wahrnehmungsform der Simultaneität zugeordnet und in ein Spannungsverhältnis zum sukzessiven Verfahren der Beschreibungspoesie gesetzt. Lessings Theorem des „bequemen Verhältnisses“ (ebd., 116) konturiert Literarizität in Abgrenzung zum außerliterarischen Sprachgebrauch. Diese Theorie ist semiolo- 
gisch fundiert. Sie formuliert die zeichentheoretische Unterscheidung zwischen den natürlichen und willkürlichen Zeichen aber in Richtung eines Medialitätsbewusstseins weiter. Denn die literarische Qualität ist nicht in der Eigenart der verwendeten Zeichen begründet, sondern in ihrer spezifischen Verwendung, also in literarischen Techniken des sinnlich gemachten Sprachgebrauchs, denen wiederum die Vermittlung einer bestimmten Qualität der Rezeption zugeordnet wird.

Die mediologische Argumentation ergänzt in Lessings Text die semiologische - mit dem Ziel, die Einzelkünste in einer bis dahin ungekannten Radikalität medial auszudifferenzieren. Eine vom Willen zur normativen Grenzziehung geleitete Medienkonkurrenz zersprengt in Lessings Programmschrift die Gleichung des ut pictura poesis-Gebots und deren Herleitung von Horaz und Simonides. „Die blendende Antithese des griechischen Voltaire, daß die Malerei eine stumme Poesie und die Poesie eine redende Malerei sei“ (ebd., 14), wird zunächst einer philologischen Kritik unterzogen, um sie dann systematisch zu widerlegen. Der Wille zur Grundsätzlichkeit der medialen Ausdifferenzierung bedingt eine Rückführung der Einzelkünste auf ihre ersten und einfachsten Parameter als mediale Bedingungen ihrer Existenz. Nur von dieser Intention her ist der in seiner Simplizität gleichermaßen geniale wie reduktionistische Gestus der Entgegensetzung von bildender Kunst als Raumkunst vs. Poesie als Zeitkunst angemessen $\mathrm{zu}$ verstehen. Mit systematischem Anspruch wird die medientheoretische Argumentation zugespitzt auf den Kardinalgegensatz, wonach „die Malerei zu ihren Nachahmungen ganz andere Mittel oder Zeichen gebrauchet als die Poesie; jene nämlich Figuren und Farben in dem Raume, diese aber artikulierte Töne in der Zeit“ (ebd., 116).

\section{„Pygmalion als Betrachter“: Hermeneutik der bildenden Kunst}

Lessings Bezugsfeld für die Aufkündigung des ut pictura poesis-Gebots und die Formulierung einer spezifischen Medienästhetik für Literatur und Bildkunst war ein doppeltes, in diskursiver Verschränkung: erstens die Aufklärungspoetik und zweitens die neue Gattung der Kunstliteratur, wie sie von Winckelmann mit seiner Programmschrift des Klassizismus, den Gedanken über die Nachahmung der griechischen Werke in Malerei und Bildhauerkunst (1755), begründet worden war. Von Moses Mendelssohn bereits 1756 auf dieses diskursbegründende Werk zur kanonischen Geltung der antiken Bildwerke für die Kunstproduktion der Gegenwart aufmerksam gemacht, sah Lessing in Winckelmanns Formulierung des Schönheitsideals als Affektdämpfung unter der Formel ,edle Einfalt und stille Größe‘ (vgl. Winckelmann 1995b [1755], 30-31) eine Beschränkung für die 
Ausdrucksmöglichkeiten der Literatur. Es ist ein Beleg für die diskursive Dominanz des Klassizismus und seines bevorzugten Paradigmas, der antiken Plastik der Menschengestalt, dass die erste moderne Medienästhetik ihre Trennschärfe gerade in Abgrenzung zur kanonischen Geltung der klassizistischen Kunstliteratur ausgebildet hat. Lessing formulierte seine medienästhetische Wendung der Kunsttheorie in Auseinandersetzung mit dem zentralen Gebot Winckelmanns, das dieser an der in den Vatikanischen Museen befindlichen hellenistischen Laokoon-Gruppe exemplifiziert hatte. Der ungehemmte Ausdruck des Schmerzes sei unvereinbar mit dem antiken Schönheitsgebot. Lessing schränkt dieses Gebot auf die Bildkünste ein, deren Gesetz die Schönheit der Körper sei, während er die Literatur von ihm ausnimmt, weil ihre Essenz Handlung, Leben und Bewegung sei. Die Formulierung des Fundamentalgegensatzes zwischen der Sukzessivität ihres medialen Vorzugs im Unterschied zur Simultaneität der Bildkunst dient somit gegen die kanonische Formulierung des klassizistischen Ideals durch Winckelmann der Rechtfertigung des Leidenschaftsausdrucks in der Literatur. Daher schreie der Laokoon der antiken Statuengruppe nicht, während die Figur des Laokoon in Vergils Aeneis ebenso ungehemmt seinen Schmerz durch Schreien ausdrücken dürfe wie die Helden Homers oder der leidende Philoktet in Sophokles’ gleichnamiger Tragödie (vgl. Lessing 1990 [1766], 17).

Auch wenn Lessings Medienästhetik die Poetik weit mehr im Blick hat als die bildende Kunst, situiert sie sich doch im Feld der von Winckelmann begründeten Form der Kunstreflexion, reagiert auf diese und wirkt gewichtig auf sie zurück. Lessings Lehre von der medialen Trennung der Künste liefert systematische Argumente für die Autonomie des Visuellen, die in der klassizistischen Kunstliteratur erstmals durch Winckelmanns neue ästhetisierte Hermeneutik der Kunstbetrachtung ins Blickfeld getreten war. Lessings Kritik an der „Allegoristerei“ (ebd., 209) in der Malerei gilt der Emanzipation der reinen Körperschönheit von gedanklichen Gehalten aus dem humanistischen und christlichen Bildungskosmos. Angelegt fand er diese Argumentation zugunsten der Befreiung des Visuellen aus der Überformung durch das Wort schon bei Winckelmann in seinen Überlegungen zur Allegorie und vor allem in seinen radikal subjektivierten, literarisierten Statuenbeschreibungen. Denn auch wenn Winckelmann nachdrücklich die Auffassung vertritt, die bildende Kunst sei auch Weisheitslehre, und insofern der humanistischen Prägung des ut pictura poesis-Gebots mit seiner Orientierung am Bildungswissen verpflichtet blieb, so zeigt sich doch in seinem Allegoriekonzept, dass der auf gedankliche Gehalte verweisende Charakter der Allegorie als eines konventionellen ikonografischen Zeichens als unkünstlerisch empfunden wird. Gerade am Problem der Versinnlichung der Allegorie und ihrer Einbindung in die selbstreferentielle Bedeutung des ästhetischen Zeichens artikuliert sich im Klassizismus ein Problemdruck, der sich bis zu Winckelmanns Spätwerk, dem 
Versuch einer Allegorie (1766), in ständigen Nachbesserungen am Konzept der Allegorie manifestiert (vgl. Pfotenhauer et al. 1995, 365-366).

Die Immanenz des Sinnlichen wird aber nicht nur konzeptuell gedacht. Sie wird literarisch evoziert durch Winckelmanns emphatische und im Ton unerhört neue Hermeneutik der antiken Plastik. Allen voran seine ambitionierte Beschreibung des „Torso vom Belvedere“ in ihren verschiedenen Fassungen hat diese ästhetisierte, den Standpunkt des Hermeneuten und die historische Distanz thematisierende Betrachtungsweise in die Kunstdebatte eingeführt. Nicht mehr die antiquarisch zu rekonstruierende römische Geschichte, sondern ein seiner religiösen und historischen Bezüge entkleideter, literarisierter griechischer Mythos ist der hermeneutische Bezugspunkt für die Ekphrasis (vgl. 4.1 WANDHOFF, 2.6 RIPPL) der fragmentarisch vorgefundenen und erst halluzinativ zu vervollständigenden Kunstdenkmäler. Sieht Winckelmann beim ersten Blick vor dem „halb vernichtet[en]“ Torso nichts als einen „ungeformten Stein“ (Winckelmann 1995a [1759], 176), so muss der hermeneutische Widerstand erst überwunden, der Steinklumpen durch Eros, assoziative Erinnerung an Homer und phantasmatische Ergänzung durch die Einbildungskraft zu lebendigem Kunsterleben transformiert werden. Bezugspunkt dieser hergestellten Präsenz ist die Gegenwart des Betrachtens, das konkrete sinnliche Erlebnis, die Autopsie des Blicks, der sich in den fließenden Linien der Muskeln verliert und von ihnen „gleichsam mit verschlungen“ wird (ebd., 177). Der Mythos dient in dieser ästhetisierten Form nicht mehr als ikonografischer Kommentar. Das konkrete sinnliche Werk entfaltet in der literarischen Beschreibung ein Eigenleben, unabhängig vom Bildungswissen (vgl. Käfer 1986, 61-93). Als Beschreibungsmodell für diese neue Art der Kunsthermeneutik dient im zeitgenössischen Diskurs der Mythos von Pygmalion aus Ovids Metamorphosen (vgl. Ovid 2007 [ca. 1-8 n. Chr.], 495-498), der um 1750 eine erstaunliche Konjunktur hat, so bei André-François Boureau-Deslandes, Jean-Jacques Rousseau, Denis Diderot, Wilhelm Heinse, Winckelmann und Herder (vgl. Neumann 1997). Die vom zyprischen Bildhauer Pygmalion geschaffene Statue, die bei Ovid von Venus erweckt wird, wird zum Sinnbild für die erotisch motivierte, phantasmatisch gesteigerte und radikal subjektivierte Betrachtungskunst. „Pygmalion als Betrachter“ - so fasst der Kunsthistoriker Oskar Bätschmann diese verlebendigende Hermeneutik in eine prägnante Formel (vgl. Bätschmann 1978).

Der Mythos von Pygmalion geht in der Kunstreflexion des ausgehenden 18. Jahrhunderts eine beziehungsreiche Synthese mit Lessings Theorem des „prägnanten Moments“ (Lessing 1990 [1766], 32) ein, wodurch die Kategorie der Illusionierung als Eigenschaft der produktiven Einbildungskraft des Betrachters näher bestimmt wird. Auch hier zeigt sich der prägende Einfluss von Lessings Medienästhetik auf die Kunstliteratur des Klassizismus wie des Antiklassizismus um 1800 (vgl. Wolf 2002). Lessing musste mit diesem Theorem auf Umwegen das Problem 
der Zeitbehandlung in der Bildkunst lösen, das sich durch deren Festlegung auf die Raumdimension und den prinzipiellen Ausschluss der Zeitfolge ergab. Auch im Falle der Bildkunst denkt Lessing in narrativen Kategorien, wenn er den dargestellten einzigen Moment der Bildkunst als zeitverdichtete Form ansieht, die das soeben Vergangene ebenso erahnen lässt wie das unmittelbar Zukünftige. Die Rückübersetzung des im Moment gebannten zeitlichen Geschehens in Ablauf und Bewegung aber leistet die Einbildungskraft des Betrachters. Daraus ergeben sich ästhetische Gesetzlichkeiten für die Art der Darstellung, welche der Einbildungskraft freien Spielraum gewähren muss. Lessings Konsequenz ist eine Trennung der ästhetischen Qualität der Einbildungskraft von bloßer Affektivität, wie sie noch Dubos' Variante der Wirkungsästhetik bestimmt hatte: „Dasjenige aber nur allein ist fruchtbar, was der Einbildungskraft freies Spiel läßt. Je mehr wir sehen, desto mehr müssen wir hinzudenken können. Je mehr wir dazudenken, desto mehr müssen wir zu sehen glauben“ (Lessing 1990 [1766], 32).

Goethe hat gegen dieses verlebendigende Paradigma der Bewegungsillusion Einspruch erhoben. In seiner Kommentierung der Übersetzung von Diderots Versuch über die Malerei (1799) nennt er den Pygmalion-Mythos „ein lüsternes Geschichtchen“, des wahren Künstlers unwürdig, und formuliert als Merksatz: „Doch dieses merke: der Fuß ist von Marmor, er verlangt nicht zu gehen, und so ist der Körper auch, er verlangt nicht zu leben“; gemäß dem Programm der Weimarer Klassizisten sieht er darin ein unzulässiges „Vermischen von Natur und Kunst“ (Goethe 1998d [1799], 569-570). So findet sich in der programmatischen Einleitung in die Propyläen (1798) auch ein versteckter Seitenhieb auf Lessings Formulierung des prägnanten Moments: „Das beste Kunstwerk [...] fesselt die Gefühle, und die Einbildungskraft, es nimmt uns unsere Willkür, wir können mit dem Vollkommenen nicht schalten und walten, wie wir wollen“ (Goethe 1998b [1798], 468). Goethes eigener Beitrag zur Laokoon-Debatte, programmatisch in den Propyläen veröffentlicht, ist daher sowohl gegen die imaginative Steigerung des Gesehenen im Theorem des ,prägnanten Moments‘ als auch gegen eine Hermeneutik des Leidenschaftsausdrucks im Zeichen gesteigerter Expressivität gerichtet. „Den Blick auf das Geschehen an der skulpturalen Oberfläche zu lenken“ (Mülder-Bach 2000, 472), und zwar im Namen einer Immanenz der ästhetischen Gestalt als selbstreferentieller Konfiguration, ist Goethes Anliegen im Aufsatz Über Laokoon (1798). Goethe denkt somit Lessings Medienästhetik weiter, modifiziert und korrigiert sie sowohl in Bezug auf ihre Gegenstandslehre als auch ihren Zentralbegriff der Illusion. Erstere, bei Lessing durch „Figuren und Körper in dem Raume“ (Lessing 1990 [1766], 116) bestimmt, wird in Goethes Aufsatz um den Faktor genuin bildnerischer Zeitlichkeit und die Erkenntnis bildnerischer Syntax und Logik ergänzt, letzterer wird durch die Autonomie des Kunstwerks gegenüber der Suggestion von Leben und Natur ersetzt. Goethe erkennt in der 
Laokoon-Gruppe ein bildnerisches Gefüge, das von einem zentralen „Lebenspunkt“ (Goethe 1994 [1797], 97), dem Biss der Schlange, in seinen Ursachen und Wirkungen in sich stimmig organisiert ist. Die Bewegtheit der Figuren ist durch diese bildnerischen Kausalitätszusammenhänge motiviert und bedarf nicht der Einbildungskraft zu ihrer Realisierung: „Es ist also dieses ein Hauptsatz: der Künstler hat uns eine sinnliche Wirkung dargestellt, er zeigt uns auch die sinnliche Ursache“ (Goethe 1998c [1798], 494).

Goethes Laokoon-Aufsatz versteht sich als programmatischer Beitrag zur Gegenstandslehre des Weimarer Klassizismus, welche die Konsequenzen der medialen Ausdifferenzierung und damit letztlich auch Lessings mediologisches Argument des „bequemen Verhältnisses“(Lessing 1990 [1766],116) zwischenästhetischem Medium und seinem Inhalt weiterdenkt. Die Befreiung des Visuellen von der Vorherrschaft des Diskursiven im Namen der Kunstautonomie hat Konsequenzen für die Auffassung des bildnerischen Gegenstands, der ohne ikonografisches Bezugsystem rein auf der Ebene der sinnlichen Erscheinung bestimmt werden will (vgl. Pfotenhauer 2004, 157-159; Osterkamp 2011a, 15-19). „Die vorteilhaftesten Gegenstände sind die, welche sich durch ihr sinnliches Dasein selbst bestimmen“, schreibt Goethe im Aufsatz Über die Gegenstände der bildenden Kunst; sie müssen sich „beim ersten Anschauen sowohl im ganzen als in ihren Theilen selbst erklären“(Goethe 1998a [1797], 441-442). Entsprechend verfährt Goethe bei seinen Bildbeschreibungen, die konsequent vom ikonografischen Kontext absehen und auf das rein menschliche Geschehen und die Bildordnung abzielen (vgl. Osterkamp 1991 und 2011, 15-19). Die semiologische Konsequenz aus dieser Immanenz der sinnlichen Gestalt zieht Moritz für die Weimarer Klassik, indem er das Kunstwerk als selbstreferentielles Zeichen bestimmt, bei dem Signifikant und Signifikat zusammenfallen. Moritz radikalisiert, beispielhaft für die Kunstliteratur der Klassik wie der Frühromantik, die Konsequenzen dieser Medientrennung für die Kunstbeschreibung. Der klassische Unsagbarkeitstopos der Kunstliteratur, demzufolge das Spezifische des Kunsterlebens nicht verbalisierbar sei, erhält bei ihm eine mediologische Zuspitzung zur Frage, „[i]n wie fern Kunstwerke beschrieben werden können“ (Moritz 1995 [1788], 372). Weil das „in sich selbst vollendete“ Bildwerk „sich durch sich selbst beschreibt“, führt die Mediendifferenz zur ihm fremd gegenüberstehenden Beschreibungssprache letztlich zum Abbruch gemeinsamer Referenz. Der künstlerische Ausdruck ist kondensierter Sinn, „der nun über allen ferneren Ausdruck durch Worte erhaben ist, welche eben da aufhören müssen, wo das ächte Kunstwerk anfängt“ (ebd., 374). Moritz zieht nicht die Konsequenz des Verstummens, sondern zeigt der aporetisch gewordenen Kunstbeschreibung Wege aus dem Dilemma auf. Sie müsse sich auf eine Formanalyse der abstrakten Bildgesetze beschränken oder aber selbst zur Kunst werden, in jedem Fall sich selbstthematisch ihrer Mediendifferenz zum Beschriebenen bewusst sein. 


\section{Ausblick: Literarische Bilderlust}

Die Kunstliteratur der Romantik greift die Anregung der Übersetzung der Kunstbeschreibung in Literatur dankbar auf. Das romantische Projekt einer verwandelnden Übersetzung der Künste ineinander, wie es das Schlegelsche Kunstgespräch Die Gemälde (1799) formuliert, hat die mediale Sensibilisierung in der Kunstliteratur zur Voraussetzung (vgl. Schlegel 1996 [1799]). Die Kunstliteratur wird im 18. Jahrhundert zum Experimentierfeld für mediologische Neubestimmungen und avanciert damit auch zur Schule für die Medienbewusstheit der Literatur (vgl. Schneider 2002, 92-94 und 115-122). Goethes Romane, die - in den Wilhelm-Meister-Texten (1795-1796 und 1829) - den anthropologischen Zusammenhang wie auch die Differenz von ,Bild' und ,Bildung' ausbuchstabieren (vgl. Pfotenhauer 2000, 45-66; Voßkamp 2004; Osterkamp 2011b; Tausch 2013) oder in den Wahlverwandtschaften (1809) - das ästhetische Risiko des Bilderzaubers (vgl. Schneider 2012, 114-123) vorführen, zeugen ebenso von diesem Zusammenhang (etwa die romantischen Poetiken der Arabeske;vgl. Oesterle 1991) wie E. T. A. Hoffmanns Fantasiestücke in Callots Manier (1814-1815) oder seine Orientierung am capriccio und anderen zeichnerischen Gattungen (vgl. Schmidt 1999; Neumann 1999). Ekphrasis, fiktive und phantasmatische Bildszenarien, sich belebende Bilder und Tableaus, die den Handlungsverlauf stillstellen, prägen die Literatur der Kunstperiode. An ihr lässt sich die Dialektik von medialer Ausdifferenzierung und intermedialen Transgressionen zwischen den Künsten zeigen (vgl. 2.7 RIPPL), die das historische Ergebnis der skizzierten Veränderungen im System der Künste im Laufe des Jahrhunderts sind. Die in der Kunstheorie reflektierte Medienkonkurrenz zwischen Sprache und Bild wird zum Ausgangspunkt für die Bilderlust der Literatur. Der Intermedialität kommt für die Modernediagnose der Literatur um 1800 daher paradigmatische Bedeutung zu.

\section{Literaturverzeichnis}

Adler, Hans. „Fundus Animae - der Grund der Seele. Zur Gnoseologie des Dunklen in der Aufklärung“. Deutsche Vierteljahrsschrift für Literaturwissenschaft und Geistesgeschichte 62.2 (1988): 197-220.

Adler, Hans. „Prägnanz - Eine Denkfigur des 18. Jahrhunderts“. Literatur und Geschichte. Festschrift für Wulf Koepke zum 70. Geburtstag. Hrsg. von Karl Menges. Amsterdam und Atlanta, GA: Rodopi, 1998. 15-34.

Bätschmann, Oskar. „Pygmalion als Betrachter. Hans Heinrich Füssli, die Niobiden und die Rezeption von Plastik und Malerei in der zweiten Hälfte des 18. Jahrhunderts“. Beiträge zur Kunst des 17. und 18. Jahrhunderts in Zürich. Hrsg. von Peter Vignau-Wilberg und Jana Hofmeister. Zürich: Schweizerisches Institut für Kunstwissenschaft, 1978. 179-198. 
Baumgarten, Alexander Gottlieb. Ästhetik. Lateinisch-Deutsch. Übers. und hrsg. von Dagmar Mirbach Bd. 1. Hamburg: Meiner, 2007 [1750-1758].

Baxmann, Inge, Michael Franz und Wolfgang Schäffner (Hrsg.). Das Laokoon-Paradigma. Zeichenregime im 18. Jahrhundert. Berlin: Akademie, 2000.

Boehm, Gottfried. „Die Wiederkehr der Bilder“. Was ist ein Bild? Hrsg. von Gottfried Boehm. München: Fink, 1994. 11-38.

Breitinger, Johann Jakob. Critische Dichtkunst. Faksimiledruck nach der Ausgabe Zürich 1740. Stuttgart: Metzler, 1966 [1740].

Buch, Hans Christoph. Ut pictura poesis. Die Beschreibungsliteratur und ihre Kritiker von Lessing bis Lukács. München: Hanser, 1972.

Dubos, Jean-Baptiste. Kritische Betrachtungen über die Poesie und Mahlerey. Übers. von Gottfried Benediktus Funk. 1. Theil. Kopenhagen: Mummische Buchhandlung 1760 [1719].

Gaier, Ulrich. „Denken als Bildprozeß. Vorstellungsart und Denkbild um 1800“. Bildersturm und Bilderflut um 1800. Zur schwierigen Anschaulichkeit der Moderne. Hrsg. von Helmut J. Schneider, Ralf Simon und Thomas Wirtz. Bielefeld: Aisthesis, 2001. 19-51.

Goethe, Johann Wolfgang von. „Aus einer Reise in die Schweiz im Jahre 1797“ [1797]. Sämtliche Werke, Briefe, Tagebücher und Gespräche Abt. 1. Sämtliche Werke. Bd.16: Campagne in Frankreich, Belagerung von Mainz, Reiseschriften. Hrsg. von Klaus-Detlef Müller. Frankfurt am Main: Deutscher Klassiker Verlag, 1994. 90-214.

Goethe, Johann Wolfgang von. „Über die Gegenstände der bildenden Kunst“ [1797]. Sämtliche Werke, Briefe, Tagebücher und Gespräche Abt. 1. Sämtliche Werke. Bd. 18: Ästhetische Schriften. 1771-1805. Hrsg. von Friedmar Apel. Frankfurt am Main: Deutscher Klassiker Verlag, 1998a. 441-444.

Goethe, Johann Wolfgang von. „Einleitung in die Propyläen“ [1798]. Sämtliche Werke, Briefe, Tagebücher und Gespräche Abt. 1. Sämtliche Werke. Bd. 18: Ästhetische Schriften.1771-1805. Hrsg. von Friedmar Apel. Frankfurt am Main: Deutscher Klassiker Verlag, 1998b. 457-475.

Goethe, Johann Wolfgang von. „Über Laokoon“ [1798]. Sämtliche Werke, Briefe, Tagebücher und Gespräche Abt. 1. Sämtliche Werke. Bd. 18: Ästhetische Schriften. 1771-1805. Hrsg. von Friedmar Apel. Frankfurt am Main: Deutscher Klassiker Verlag, 1998c. 489-500.

Goethe, Johann Wolfgang von. „Diderots Versuch über die Malerei“ [1799]. Sämtliche Werke, Briefe, Tagebücher und Gespräche Abt. 1. Sämtliche Werke. Bd. 18: Ästhetische Schriften. 1771-1805. Hrsg. von Friedmar Apel. Frankfurt am Main: Deutscher Klassiker Verlag, 1998d. 559-608.

Horaz [Quintus Horatius Flaccus]. „De arte poetica liber/Das Buch von der Dichtkunst“ [14 v. Chr.]. Sämtliche Werke. Lateinisch und Deutsch. Teil I nach Kayser, Nordenflycht, Burger hrsg. von Hans Färber, Teil II übers. und bearb. von Hans Färber und Wilhelm Schöne. Darmstadt: Wissenschaftliche Buchgesellschaft, 1967. 230-259.

Jakob, Ludwig Heinrich. Grundriß der Erfahrungs-Seelenlehre. Halle: Hemmerde \& Schwetschke, 1791.

Käfer, Markus. Winckelmanns hermeneutische Prinzipien. Heidelberg: Winter, 1986.

Kant, Immanuel. „Anthropologie in pragmatischer Hinsicht“ [1798]. Werkausgabe Bd. 12: Schriften zur Anthropologie, Geschichtsphilosophie, Politik und Pädagogik 2. Register zur Werkausgabe. Hrsg. von Wilhelm Weischedel. Frankfurt am Main: Suhrkamp, 1977a. 399-694.

Kant, Immanuel. Werkausgabe Bd. 10: Kritik der Urteilskraft. Hrsg. von Wilhelm Weischedel. Frankfurt am Main: Suhrkamp, 1977b [1790]. 
Koebner, Thomas (Hrsg.). Laokoon und kein Ende. Der Wettstreit der Künste. München: edition text + kritik, 1989.

Koschorke, Albrecht. Körperströme und Schriftverkehr. Mediologie des 18. Jahrhunderts. München: Fink, 1999.

Leibniz, Gottfried Wilhelm. „Meditationes De Cognitione, Veritate, et Ideis, per G. G. L.“ [1684]. Sämtliche Schriften und Briefe. Reihe 6: Philosophische Schriften. Bd. 4: 1677-Juni 1690. Teil A. Hrsg. von der Leibniz-Forschungsstelle der Universität Münster. Berlin: Akademie, 1999. 585-592.

Lessing, Gotthold Ephraim. „Laokoon“ [1766]. Werke und Briefe Bd. 5.2: Werke 1766-1769. Hrsg. von Wilfried Barner. Frankfurt am Main: Deutscher Klassiker Verlag, 1990. 9-321.

Mendelssohn, Moses. „Über die Hauptgrundsätze der schönen Künste und Wissenschaften“ [1757]. Ästhetische Schriften in Auswahl. Hrsg. von Otto F. Best. 2. Aufl. Darmstadt: Wissenschaftliche Buchgesellschaft. 1986. 173-197.

Moritz, Karl Philipp. „Die Signatur des Schönen/In wie fern Kunstwerke beschrieben werden können?“ [1788]. Bibliothek der Kunstliteratur Bd. 3: Klassik und Klassizismus. Hrsg. von Helmut Pfotenhauer und Peter Sprengel. Frankfurt am Main: Deutscher Klassiker Verlag, 1995. 372-383.

Mülder-Bach, Inka. „Sichtbarkeit und Lesbarkeit. Goethes Aufsatz Über Laokoon“. Das Laokoon-Paradigma. Zeichenregime im 18. Jahrhundert. Hrsg. von Inge Baxmann, Michael Franz und Wolfgang Schäffner. Berlin: Akademie, 2000. 465-479.

Neumann, Gerhard. „Pygmalion. Metamorphosen des Mythos“. Pygmalion. Die Geschichte des Mythos in der abendländischen Literatur. Hrsg. von Matthias Mayer und Gerhard Neumann. Freiburg im Breisgau: Rombach, 1997. 11-60.

Neumann, Gerhard. „Narration und Bildlichkeit. Zur Inszenierung eines romantischen Schicksalsmusters in E. T. A. Hoffmanns Novelle Doge und Dogaresse“. Bild und Schrift in der Romantik. Hrsg. von Gerhard Neumann und Günter Oesterle. Würzburg: Königshausen \& Neumann, 1999. 107-142.

Neumann, Gerhard, und Günter Oesterle. „Einleitung“. Bild und Schrift in der Romantik. Hrsg. von Gerhard Neumann und Günter Oesterle. Würzburg: Königshausen \& Neumann, 1999. 9-23.

Oesterle, Günter. „Arabeske, Schrift und Poesie in E. T. A. Hoffmanns Kunstmärchen Der goldene Topf'. Athenäum. Jahrbuch für Romantik 1 (1991): 69-107.

Osterkamp, Ernst. Im Buchstabenbilde. Studien zum Verfahren Goethescher Bildbeschreibungen. Stuttgart: Metzler, 1991.

Osterkamp, Ernst. „Goethes Beschäftigung mit den bildenden Künsten. Ein werkbiographischer Überblick“. Goethe-Handbuch. Supplemente Bd. 3: Kunst. Hrsg. von Andreas Beyer und Ernst Osterkamp. Stuttgart und Weimar: Metzler, 2011a. 3-27.

Osterkamp, Ernst. „Kunst und Künstler in Goethes dichterischem Werk“. Goethe-Handbuch. Supplemente Bd. 3: Kunst. Hrsg. von Andreas Beyer und Ernst Osterkamp. Stuttgart und Weimar: Metzler, 2011b. 197-209.

Ovid. Metamorphosen. Übers. und hrsg. von Gerhard Fink. 2. Aufl. Düsseldorf: Artemis \& Winkler, 2007 [ca. 1-8 n. Chr.].

Pethes, Nicolas. „Intermedialitätsphilologie? Lichtenbergs Textmodell und der implizite Mediendiskurs der Literatur“. Deutsche Vierteljahrsschrift für Literaturwissenschaft und Geistesgeschichte 76.1 (2002): 86-104.

Pfeiffer, Ludwig K. Das Mediale und das Imaginäre. Dimensionen kulturanthropologischer Medientheorie. Frankfurt am Main: Suhrkamp, 1999. 
Pfotenhauer, Helmut. „Die Signatur des Schönen oder In wie fern Kunstwerke beschrieben werden können? Zu Karl Philipp Moritz und seiner italienischen Ästhetik“. Kunstliteratur als Italienerfahrung. Hrsg. von Helmut Pfotenhauer. Tübingen: Niemeyer, 1991. 67-83.

Pfotenhauer, Helmut. Sprachbilder. Untersuchungen zur Literatur seit dem achtzehnten Jahrhundert. Würzburg: Königshausen \& Neumann, 2000.

Pfotenhauer, Helmut. „,Weimarer Klassik“ als Kultur des Sichtbaren“. Begrenzte Natur und Unendlichkeit der Idee. Literatur und Bildende Kunst in Klassizismus und Romantik. Hrsg. von Jutta Müller-Tamm und Cornelia Ortlieb. Freiburg im Breisgau: Rombach, 2004. $145-182$.

Pfotenhauer, Helmut, Markus Bernauer und Norbert Miller (Hrsg.). Bibliothek der Kunstliteratur Bd. 2: Frühklassizismus. Position und Opposition: Winckelmann, Mengs, Heinse. Frankfurt am Main: Deutscher Klassiker Verlag, 1995.

Plutarch. „Ob die Athener im Kriege oder in der Weisheit berühmter waren“ [1. Jh. n. Chr.]. Moralia Bd. 1. Übers. von J. C. F. Bähr. Hrsg. von Christian Weise und Manuel Vogel. Wiesbaden: Marix, 2012. 601-611.

Schlegel, August Wilhelm von. Die Gemählde. Gespräch. Hrsg. von Lothar Müller. Amsterdam und Dresden: Verlag der Kunst, 1996 [1799].

Schmidt, Olaf. „Die Wundernadel des Meisters“ - Zum Bild-Text-Verhältnis in E. T. A. Hoffmanns Capriccio ,Prinzessin Brambilla‘. E. T. A. Hoffmann-Jahrbuch (1999): 29-49.

Schneider, Helmut J., Ralf Simon und Thomas Wirtz (Hrsg.). Bildersturm und Bilderflut um 1800. Zur schwierigen Anschaulichkeit der Moderne. Bielefeld: Aisthesis, 2001.

Schneider, Sabine. „Klassizismus und Romantik - Zwei Konfigurationen der einen ästhetischen Moderne. Konzeptuelle Überlegungen und neuere Forschungsperspektiven“. Jahrbuch der Jean-Paul-Gesellschaft 37 (2002): 86-128.

Schneider, Sabine. „,Wilde Semiose‘. Kontaminierte Zeichen und infektiöse Bilder bei Goethe und Kleist“. Kultur-Schreiben als romantisches Projekt. Romantische Ethnographie im Spannungsfeld zwischen Imagination und Wissenschaft. Hrsg. von David E. Wellbery. Würzburg: Königshausen \& Neumann. 2012.105-134.

Simon, Ralf. Der poetische Text als Bildkritik. München: Fink, 2009.

Stierle, Karlheinz. „Das bequeme Verhältnis. Lessings Laokoon und die Entdeckung des ästhetischen Mediums“. Das Laokoon-Projekt. Pläne einer semiotischen Ästhetik. Hrsg. von Gunter Gebauer. Stuttgart: Metzler, 1984. 23-58.

Tausch, Harald. „Architektur und Bild in Goethes Roman Wilhelm Meisters Wanderjahre (1821)“. Zwischen Architektur und literarischer Imagination. Hrsg. von Andreas Beyer, Ralf Simon und Martino Stierli. München: Fink, 2013. 275-315.

Voßkamp, Wilhelm. Ein anderes Selbst. Bild und Bildung im deutschen Roman des 18. und 19. Jahrhunderts. Göttingen: Wallstein. 2004.

Wackenroder, Wilhelm Heinrich. „Von zwei wunderbaren Sprachen und deren geheimnisvoller Kraft“ [1796]. Sämtliche Werke und Briefe Bd. 2: Werke. Hrsg. von Silvio Vietta. Heidelberg: Winter. 1991. 97-100.

Willems, Gottfried. Anschaulichkeit. Zu Theorie und Geschichte der Wort-Bild-Beziehungen und des literarischen Darstellungsstils. Tübingen: Niemeyer, 1989.

Winckelmann, Johann Joachim. „Beschreibung des Torso im Belvedere zu Rom“ [1759]. Bibliothek der Kunstliteratur Bd. 2: Frühklassizismus. Position und Opposition: Winckelmann, Mengs, Heinse. Hrsg. von Helmut Pfotenhauer, Markus Bernauer und Norbert Miller. Frankfurt am Main: Deutscher Klassiker Verlag, 1995a. 174-180. 
Winckelmann, Johann Joachim. „Gedanken über die Nachahmung der griechischen Werke in der Malerei und Bildhauerkunst“ [1755]. Bibliothek der Kunstliteratur Bd. 2: Frühklassizismus. Position und Opposition: Winckelmann, Mengs, Heinse. Hrsg. von Helmut Pfotenhauer, Markus Bernauer und Norbert Miller. Frankfurt am Main: Deutscher Klassiker Verlag, 1995b. 9-50.

Wolf, Norbert Christian. „,Fruchtbarer Augenblick‘ - ,prägnanter Moment‘: Zur medienspezifischen Funktion einer ästhetischen Kategorie in Aufklärung und Klassik (Lessing, Goethe)“. Prägnanter Moment. Studien zur deutschen Literatur der Aufklärung und Klassik. Festschrift für Hans-Jürgen Schings. Hrsg. von Peter-André Alt, Alexander Košenina, Hartmut Reinhard und Wolfgang Riedel. Würzburg: Königshausen \& Neumann, 2002. 373-404. 